\title{
1 Symmetry and Shannon Measure of Ordering: Paradoxes 2 of Voronoi Tessellation
}

\author{
Edward Bormashenko, Irina Legchenkova, Mark Frenkel \\ 1 Department of Chemical Engineering, Biotechnology and Materials, Engineering Sciences Faculty, Ariel \\ University, Ariel, Israel 407000 \\ * Correspondence: Dr. Edward Bormashenko: edward@ariel.ac.il ; Tel.: +972 0747296863 \\ Dr. Irina Legchenkova: ilegchenkova@gmail.com ; Dr. Mark Frenkel: markfr@ariel.ac.il \\ Received: date; Accepted: date; Published: date
}

\begin{abstract}
Voronoi entropy for the random patterns and patterns demonstrating various elements of symmetry are calculated. The symmetric patterns are characterized by the values of the Voronoi entropy very close to those inherent to random ones. This contradicts the idea that the Voronoi entropy quantifies the ordering of the seed points, constituting the pattern. The extension of the Shannon-like formula embracing symmetric patterns is suggested. Analysis of Voronoi diagrams enables revealing of the elements of symmetry of the pattern.
\end{abstract}

Keywords: Voronoi entropy; Voronoi tessellation; symmetry; ordering; Shannon measure of information

\section{Introduction}

Symmetry considerations play a key role in modern science [1-3], giving rise to the conservation laws in physics, and being dominating in quantum theory [4], crystallography [5], condensed-matter physics [6], thermodynamics, chemistry and biology [1-3]. Moreover, symmetry considerations are of a fundamental importance in aesthetics and science-art relations [1, 7-8]. The other fundamental value playing the central place in modern science is the Shannon measure of information [9-14]. In the present article we demonstrate that the analysis of the Voronoi diagrams (or Voronoi tessellations) enables the synthesis of symmetry and the Shannon measure of ordering considerations [15-18]. Voronoi diagrams arise from the problems involving patterns with a surface distribution of spots [19]. A Voronoi tessellation (or diagram or mosaic) is a partitioning of the plane into regions based on the distance to a specified discrete set of points (called seeds, sites, nuclei, or generators) [17,18]. For each seed, there is a corresponding region consisting of all points closer to that seed than to any other. The Voronoi polyhedron of a point nucleus in space is the smallest polyhedron formed by the perpendicularly bisecting planes between a given nucleus and all the other nuclei. The Voronoi tessellation divides a region into space-filling, non-overlapping convex polyhedrons [17-20]. It was already mentioned, that the idea of what is now called the Voronoi tessellation has been proposed already by Johannes Kepler and Rene Descartes in the 17th century [16,18, 21]. Kepler used it to study the densest sphere packing problem, whereas Descartes employed these tessellations to verify that the distribution of matter in the Universe forms vortices centered at fixed stars [21]. 
It is generally accepted that the Voronoi diagrams allow to quantify the orderliness of the $2 \mathrm{D}$ distribution of points, with the so-called Voronoi entropy, defined according to the Shannon-like formula as:

$$
S_{\text {vor }}=\sum_{i} P_{i} \ln P_{i}
$$

where $P_{i}$ is the fraction of polygons with $k$ sides or edges (also called the coordination number of the polygon) in a given Voronoi diagram [10-12]. The summation in Eq. 1 is performed from $k=3$ to the largest coordination number of any available polygon, e.g., to $k=6$ if a polygon with the largest number of edges is a hexagon. In our recent paper we demonstrated that labeling of the value $S_{v o r}$ with the wording "Voronoi entropy" is misleading and confusing [22]. Actually, Eq. 1 quantifies the average Shannon measure of ordering of a given 2D pattern [9-14, 22]. Now we show that the situation becomes even more complicated when the given pattern demonstrates the elements of symmetry (centers, axes). In this case the Shannon measure of ordering calls for re-definition.

\section{Results and discussion}

\subsection{D Patterns Demonstrating Symmetry and their Voronoi Tessellations}

We analyzed Voronoi diagrams built for sets of points demonstrating different elements of symmetry (namely centers and axes of symmetry and the rotational symmetry). At the first stage, the random sets of 200 and 1000 points located inside a circle of a given diameter were generated the by MATLAB software script. The Voronoi tessellation for the set is depicted in Figure 1. The Voronoi Entropy was calculated with the moduli of the software developed at the Department of Physics and Astronomy at the University of California (Department of Physics and Astronomy University of California, Irvine) (https://www.physics.uci.edu/ foams/do_all.html). The procedure was repeated 20 times and the averaged value of the Voronoi entropy and its standard deviation were established as $S_{v o r}=1.66 \pm 0.05$ for the set of 200 and as $S_{\text {vor }}=1.68 \pm 0.02$ for the set of 1000 points (seeds). This value is close to the Voronoi Entropy $S_{v o r}=1.71$ reported for random point patterns by other groups [23, 24]. It is reasonable to relate the difference between our calculations and the results reported in refs. 23, 24 to the boundary effects, occurring at the circumference of the pattern (see Figure 1). These random patterns will be called below the "initial patterns".

At the second stage the mirror images of the twenty of initial patterns were built and placed on the initial patterns, as shown in Figure 2. Then, their Voronoi entropy was calculated (the number of points in the new diagrams was twice of the initial ones while the area remained the same). The averaged value of the Voronoi Entropy was established as $S_{v o r}=1.64 \pm 0.05$ for a set of 200 and as $S_{v o r}=1.68 \pm 0.01$ for set of 1000 seed points. The difference between the values of the Voronoi Entropy of the initial and "mirror reflected" images was within the statistical uncertainty of the calculation, thus we conclude that the mirror reflection of the initial patterns did not change their Voronoi Entropy.

At the third stage the point symmetry (inverse) images of the twenty of initial patterns were built and put on the initial patterns, and their Voronoi entropy was calculated (again the entire number of points in the new Voronoi mosaics were twice of the initial ones). The averaged value of the Voronoi Entropy inherent for patterns similar to those depicted in Figure 3, was established as $S_{v o r}=1.66 \pm 0.06$ for a set of 200 and as $S_{\text {vor }}=1.67 \pm 0.02$ for set of 1000 points. 
At the fourth stage the initial images were rotated consequently for the angles of: $\frac{\pi}{3} ; \frac{2 \pi}{3} ; \pi ; \frac{4 \pi}{3} ; \frac{5 \pi}{3} \mathrm{rad}$ as shown in Figure 4, and the pattern demonstrating the six-fold symmetry built of 1200 points was created. The averaged value of the Voronoi entropy of these mosaics was established as $S_{v o r}=1.65 \pm 0.07$ for a set of 200 and as $S_{v o r}=1.67 \pm 0.02$ for set of 1000 points.

So we conclude that the studied symmetry transformations did not change the Voronoi value of the patterns. And this is true for the inversion, mirror reflection and the rotational symmetry operations. This result is quite trivial, as summarized in Table 1 . Indeed, the ratio of $n$-polygons in the initial patterns and in the transformed ones remains the same. Thus, the value of the Voronoi entropy, calculated according to the Eq. 1 is also expected to be the same, as demonstrated in Figure 5. However, the patterns depicted in Figures 2-4 looks definitely much more "ordered" than that of the initial random pattern, shown in Figure 1. And if we adopt that the Voronoi entropy is a true measure of ordering of 2D patterns, its value established for symmetric, "ordered" patterns, depicted in Figures 2-4 is expected to be much lower than the Voronoi entropy of the initial random mosaic, shown in Figure 1. The paradox could not be resolved by the suggestion that the symmetric mosaics shown in Figures 2-4, contain the larger number of points. Recall that the Voronoi entropy is the intensive parameter of the pattern, and it is independent of the number of points or their density (when the "boundary effects" are neglected). For example, the Voronoi entropy of the pattern built only of squares (or other identical polygons); equals zero, whatever is the number (or density) of points constituting the pattern. The Voronoi entropy of the random pattern tends to 1.71 , whatever is the number/density of seed points.

The Voronoi entropy does not change dramatically under the symmetry transformations because almost all random patterns already appear over the initial 2D distribution of points, shown in Figure 1. Mirroring or other symmetry operations, which does not change the ratio of polygons will keep the entropy almost constant. And it is not exactly constant due to the "boundary effects".

In order to resolve the puzzle and to quantify ordering appearing in various patterns we propose to re-define the generalized Voronoi Entropy for all kinds of patterns (symmetric and non-symmetric ones) as follows:

$$
S_{v o r}^{g e n}=\frac{S_{v o r}}{N}=\frac{S_{v o r}}{n+1}
$$

where $S_{v o r}$ is calculated according to Eq.1, $N$ - is the total number of the symmetry elements inherent for the pattern, including the trivial symmetry operation (in other words the rotation of the pattern to $2 \pi \mathrm{rad}$ ), and $n$ is the number of the non-trivial symmetry operations, inherent for the pattern. Eq. 2 contains Eq. 1 as a particular case; indeed, when $N=1$ and $n=0$, the 2D pattern has the trivial axis of symmetry only and we return to Eq. 1, namely: $S_{v o r}^{g e n}=S_{v o r}=\sum_{i} P_{i} \ln P_{i}$. However, for the symmetric patterns, such as those depicted in Figures 2-4, the generalized Voronoi entropy, supplied by Eq. 2, is twice smaller $(N=2)$ than that calculated for the random mosaics, shown in Figure 1. Hence, the suggested Eq. 2 quantifies better the "ordering" of 2D patterns, than the "standard" Eq. 1.

\subsection{Revealing of Symmetry in 2D patterns with Voronoi Diagrams}


114

115

116

117

118

119

120

121

122

123

124

125

126

127

128

129

130

131

132

133

134

135

136

137

138

139

140

141

142

143

144

145

146

147

148

Now consider the arbitrary 2D pattern containing $p$ points. Consider following question: does it demonstrate the elements of symmetry? We show that the Voronoi tessellation may help to answer this question. At the first stage we construct the Voronoi diagram of the pattern. Edges and vortices of the diagram form the simply connected graph. The number of edges $E$ of the graph is given by the Euler formula:

$$
E=P+V-\chi
$$

where $P$ is the number of Voronoi polygons, $V$ is the number of the vertices of the graph (in other words of the Voronoi diagram), and $\chi=2$ is the Euler characteristics of the surface. Now we sort the edges of the graph according to their length $L$. The number of edges (say $n$ of them) may have the same length $L$. Thus, the probability to find the edge with the length $L$ is given by: $P_{L}=\frac{n(L)}{E}$. Let us define the Voronoi entropy of the graph as follows:

$$
S_{\text {vor }}^{\text {graph }}=-\sum_{i} P_{L i} \ln P_{L i}
$$

If all of the edges constituting the graph are of the different lengths, its Voronoi Entropy equals $S_{\text {vor }}^{\text {graph }}=$ $\ln E$, and this means that the pattern demonstrates no symmetry. However, when the Voronoi diagram demonstrates the elements of symmetry, the edges of the same lengths appear at the Voronoi tessellation, and the Voronoi entropy of the graph, given by Eq.4, should be necessarily decreased. For example, if the Voronoi diagram has the mirror symmetry (in other words it has the axis of symmetry of second order), the simple calculation according to the Eq. 4 yields $S_{\text {vor }}^{\text {graph }}=\ln \frac{E}{2}$. The formula is easily generalized for the single axis of symmetry with an order of $m$, namely: $S_{\text {vor }}^{\text {graph }}=\ln \frac{E}{m} ; m=\operatorname{Eexp}\left(-S_{\text {vor }}^{\text {graph }}\right)$. Thus, we conclude that the Voronoi entropy of the graph is sensitive to the symmetry of the initial pattern, and enables revealing of the elements of symmetry of the pattern (or their absence); thus, making possible establishment of their number $N$ appearing in Eq. 2. The procedure enabling finding out of elements of symmetry is presented in Figure 6. Consider, that the suggested procedure does not enable establishment of $N$, defined as the total number of the symmetry elements inherent for the pattern, appearing in Eq. $\mathbf{2}$.

\section{Conclusions}

It is generally agreed that the Shannon-shaped Voronoi entropy quantifies ordering of 2D point patterns $[17,25]$. We already mentioned that labeling of the averaged Shannon measure of ordering by the notion of the "Voronoi entropy" is confusing, due to the fact that it has no relation to the thermodynamic entropy [22]. In the present paper we demonstrate that involving the traditional Shannon-like formula for characterization of ordering in symmetric patterns results in misleading estimations. It turns out that the Voronoi entropy of random patterns equals to that of symmetric ones, possessing centers or axes of symmetry, which are apparently more ordered than random mosaics. The traditional expression for the Voronoi entropy works well for non-symmetric patterns. In order to quantify ordering inherent for all kind of patterns we suggest the expression applicable for symmetrical and non-symmetrical point patterns. Analysis of the Voronoi tessellations enables finding out the elements of symmetry of the pattern. 
149 Acknowledgements: The authors gratefully acknowledge the Russian Science Foundation (project 19-19150 00076) for the financial support of this work.

151 Conflict of interests: The authors declare zero conflict of interests. 


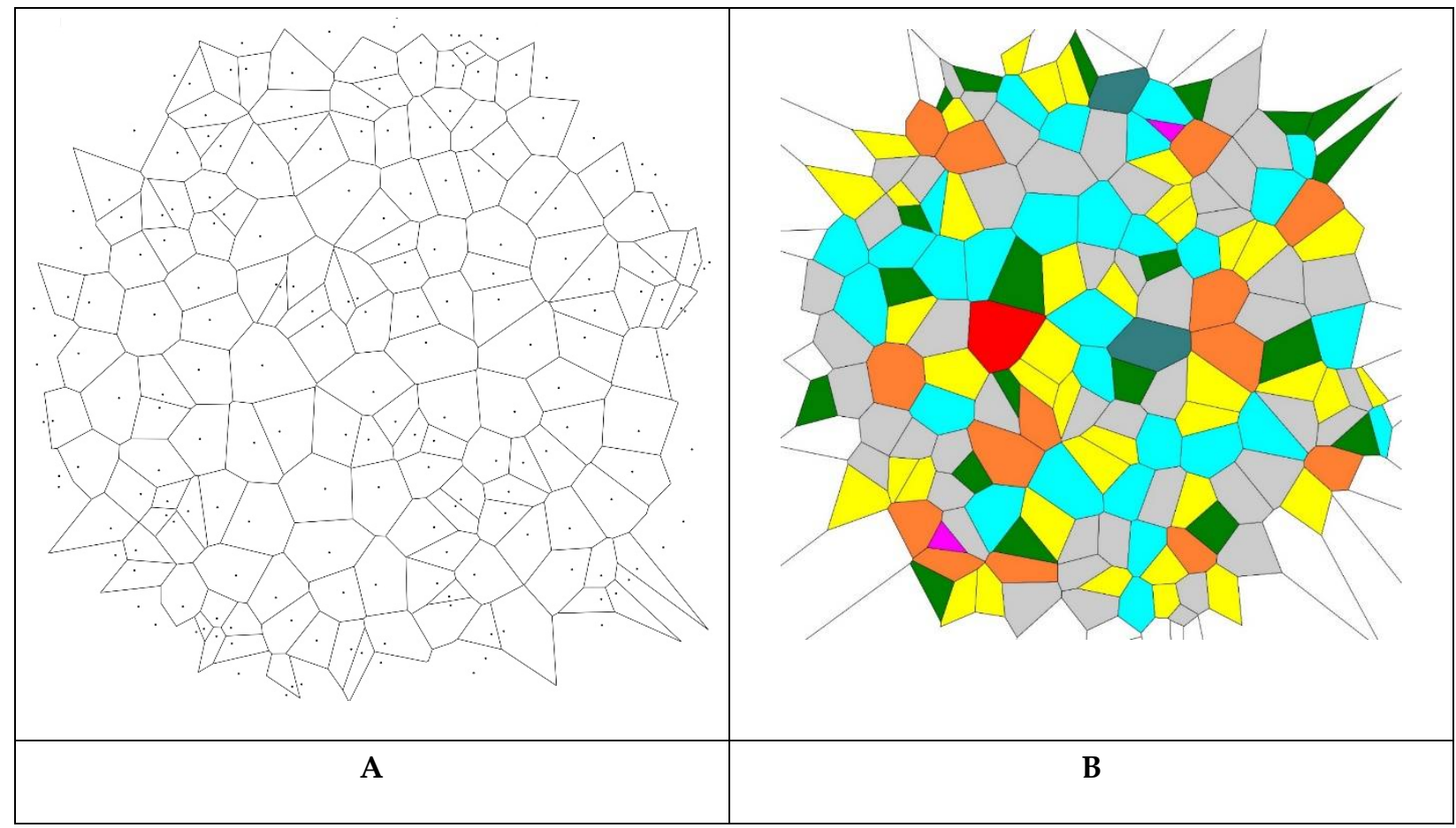

Figure 1. The Voronoi tessellation for the set of 200 random points is depicted. The Voronoi entropy is $S_{\text {vor }}=1.65$. A) The initial set of points generating the Voronoi tessellation is shown B) Colored Voronoi polygons.

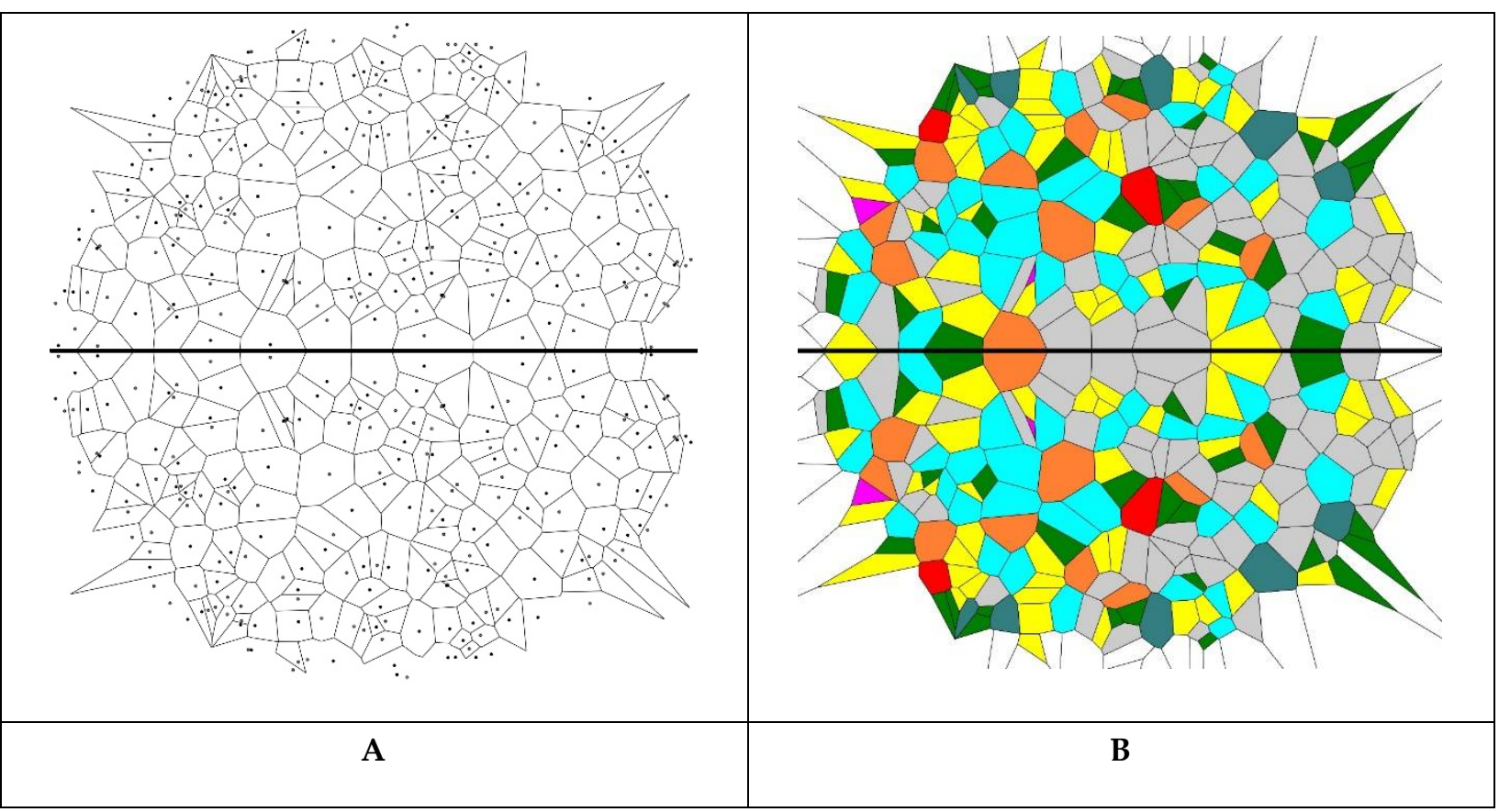

Figure 2. The mirror image of the Voronoi diagram depicted in Figure 1 is shown. The value of the Voronoi entropy is $S_{\text {vor }}=1.68$. A) Set of points generating the Voronoi construction is depicted. B) Colored 


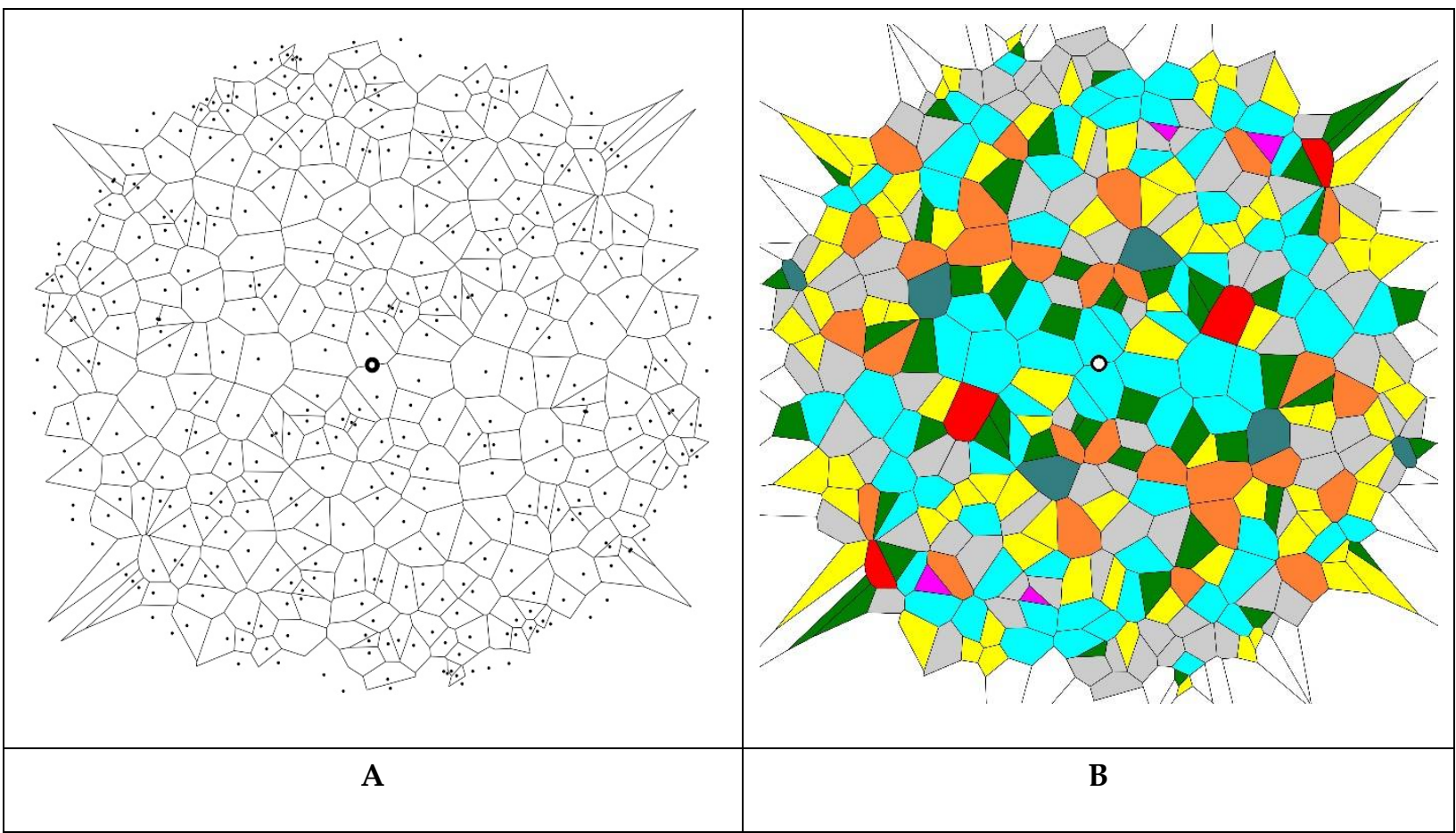

Figure 3. The point symmetry (inverse) image of the pattern, depicted in Figure $\mathbf{1}$ is shown. The value of the Voronoi entropy is $S_{v o r}=1.69$. A) Set of points generating the Voronoi tessellation is depicted. B) Colored Voronoi polygons are shown.

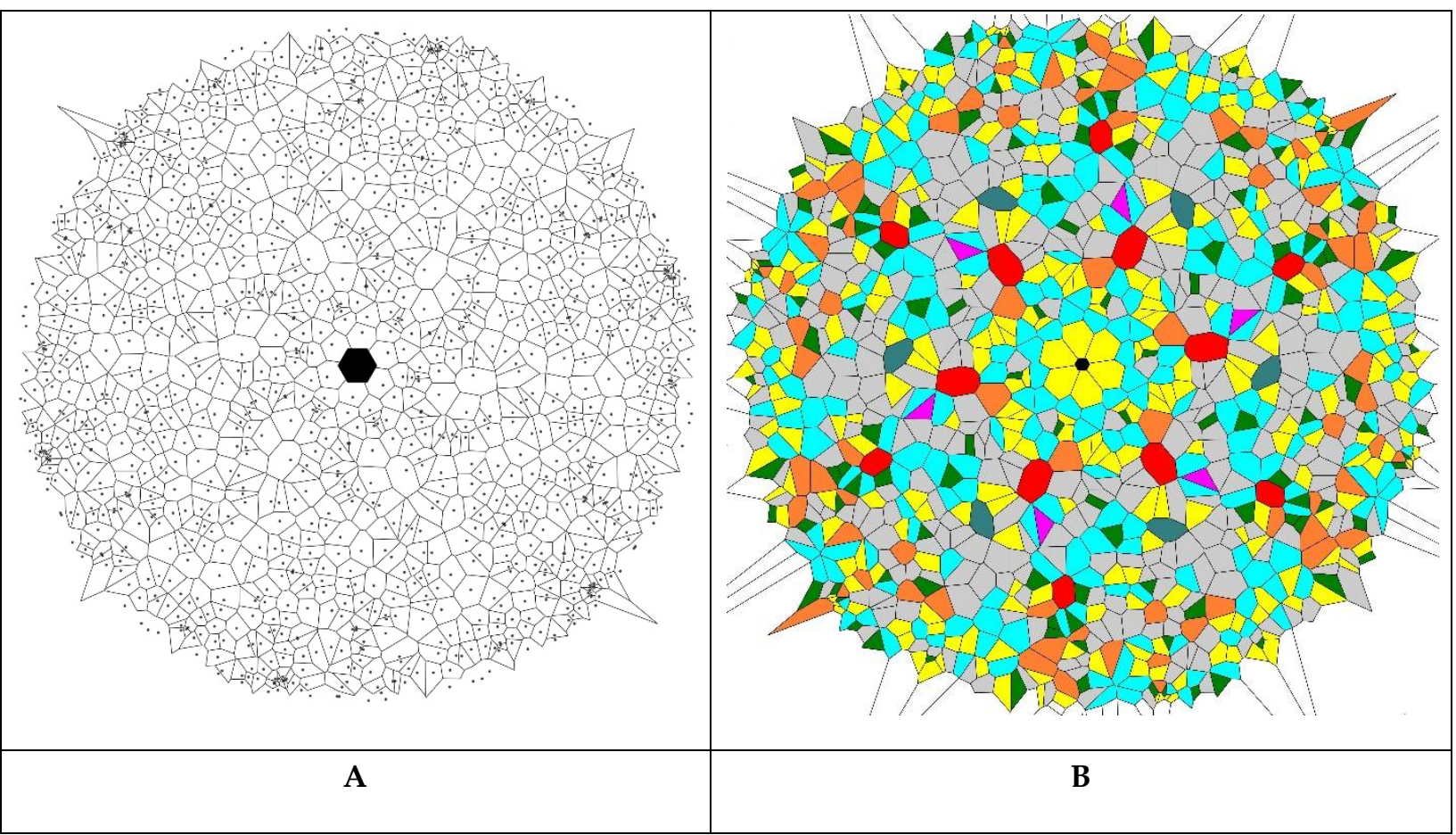

Figure 4. Six-fold symmetry pattern obtained by the rotation of the initial pattern shown in Figure 1 is depicted. The value of the Voronoi entropy is $S_{v o r}=1.57$. A) Set of points generating the Voronoi tessellation is presented. B) Colored Voronoi polygons are shown. 
167

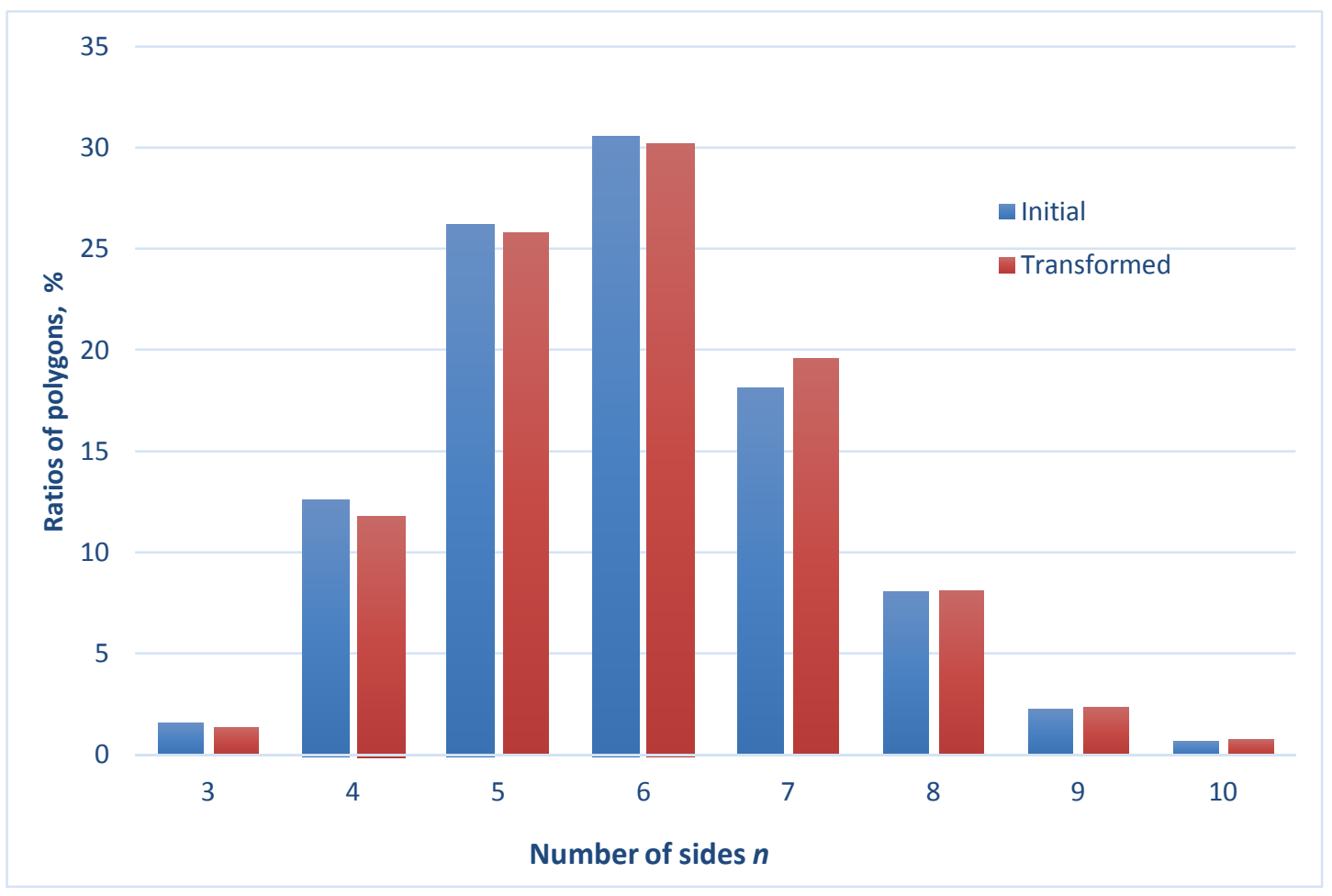

169

Figure 5. The ratios of different kinds of polygons in the initial (random) and inverse patterns are shown. 
171

172

173

174

175

176

177

178

179

180

181

182

183

184

185

186

187

188

189

190

191

192

193

194

195

196

197

198

199

200

201

202

203

204

205

\section{Construction of the Voronoi diagram}

Establishment of $E$, Calculation $S_{\text {vor }}^{\text {graph }}=-\sum_{i} P_{L i} \ln P_{L i}$

$S_{\text {vor }}^{\text {graph }}=\ln E ;$ no symmetry

$S_{\text {vor }}^{\text {graph }}<\ln E$; symmetry is present

Figure 6. The algorithm enabling finding out the elements of symmetry for a given pattern of seed points. 
206 Table 1. Voronoi entropy with its standard deviation calculated for different sets of points.

\begin{tabular}{|c|c|c|c|c|c|}
\hline & $\begin{array}{l}\text { Voronoi } \\
\text { Entropy }\end{array}$ & $\begin{array}{l}\text { Standard } \\
\text { deviation }\end{array}$ & & $\begin{array}{l}\text { Voronoi } \\
\text { Entropy }\end{array}$ & $\begin{array}{l}\text { Standard } \\
\text { deviation }\end{array}$ \\
\hline $\begin{array}{l}\text { Initial set of points (200 } \\
\text { random points) }\end{array}$ & 1.66 & \pm 0.05 & $\begin{array}{c}200 \text { random } \\
\text { points set }\end{array}$ & 1.66 & \pm 0.05 \\
\hline Mirror reflection (400 points) & 1.64 & \pm 0.05 & \multirow{2}{*}{$\begin{array}{c}400 \text { random } \\
\text { points set }\end{array}$} & \multirow{2}{*}{1.66} & \multirow{2}{*}{ \pm 0.04} \\
\hline Point reflection (400 points) & 1.66 & \pm 0.06 & & & \\
\hline $\begin{array}{l}\text { 6-fold rotational symmetry } \\
\text { (1200 points) }\end{array}$ & 1.65 & \pm 0.07 & $\begin{array}{c}1200 \text { random } \\
\text { points set }\end{array}$ & 1.68 & \pm 0.02 \\
\hline $\begin{array}{l}\text { Initial set of points (1000 } \\
\text { random points) }\end{array}$ & 1.68 & \pm 0.02 & $\begin{array}{c}1000 \text { random } \\
\text { points set }\end{array}$ & 1.68 & \pm 0.02 \\
\hline Mirror reflection (2000 points) & 1.68 & \pm 0.01 & \multirow{2}{*}{$\begin{array}{c}2000 \text { random } \\
\text { points set }\end{array}$} & \multirow{2}{*}{1.67} & \multirow{2}{*}{ \pm 0.01} \\
\hline Point reflection (2000 points) & 1.67 & \pm 0.02 & & & \\
\hline $\begin{array}{l}\text { 6-fold rotational symmetry } \\
\text { (6000 points) }\end{array}$ & 1.67 & \pm 0.02 & $\begin{array}{c}6000 \text { random } \\
\text { points set }\end{array}$ & 1.68 & \pm 0.01 \\
\hline
\end{tabular}


209

210

211

212

213

214

215

216

217

218

219

220

221

222

223

224

225

226

227

228

229

230

231

232

233

234

235

236

237

238

239

240

241

242

243

244

245

246

247

248

\section{References}

1. Weyl, H. Symmetry, Princeton University Press, Princeton, NJ, USA, 1989.

2. Van Fraassen, B. C. Laws and Symmetry, Oxford University Press, Oxford, UK, 1989.

3. Rosen, J. Symmetry in Science: An Introduction to the General Theory, Springer, Germany, 1995.

4. Hall, B. C. Quantum Theory for Mathematicians. Graduate Texts in Mathematics. Springer, 2013.

5. Chatterjee, S. K. Crystallography and the World of Symmetry, Springer, Ge, 2008.

6. El-Batanouny, M., Wooten, F. Symmetry and Condensed Matter Physics - A Computational Approach, Cambridge University Press, Cambridge, UK, 2010.

7. Darvas G., Symmetry: Cultural-historical and Ontological Aspects of Science-Arts Relations, Birkhauser, Basel, Switzerland, 2007.

8. Hargittai, I., Pickover, C.A. Spiral Symmetry, World Scientific, Singapore, 1992.

9. Shannon, C.E. A Mathematical Theory of Communication. Bell Syst. Tech. J. 1948, 27, 379-423.

10. Ben Naim, A. Shannon's Measure of information and Boltzmann's H-Theorem. Entropy 2017, 19, 48.

11. Ben-Naim, A. Information Theory; World Scientific: Singapore, 2017.

12. Ben-Naim, A. A Farewell to Entropy: Statistical Thermodynamics Based on Information; World Scientific: Singapore, 2008.

13. Ben Naim, A. An Informational Theoretical Approach to the Entropy of Liquids and Solutions, Entropy, 2018, 20(7), 514; https://doi.org/10.3390/e20070514

14. Ben-Naim, A. Entropy, the Truth the Whole Truth and Nothing but the Truth; World Scientific: Singapore, 2016.

15. Voronoi, G. Nouvelles applications des paramètres continus à la théorie des formes quadratiques. Deuxième mémoire. Recherches sur les paralléloèdres primitifs. Reine Angew. Math. 1908, 134, 198-287.

16. Liebling, T.M.; Pournin, L. Voronoi diagrams and Delaunay triangulations: Ubiquitous Siamese Twins. Doc. Math. 2012, ISMP, 419-431.

17. Barthélemy, M. Spatial networks. Phys. Rep. 2011, 499, 1-101.

18. Bormashenko, E.; Frenkel, M.; Vilk, A.; Legchenkova, I.; Fedorets, A.; Aktaev, N.; Dombrovsky, L.; Nosonovsky, M. Characterization of self-assembled 2D patterns with Voronoi Entropy. Entropy 2018, 20, 956.

19. Fedorets, A.A.; Frenkel, M.; Bormashenko, E.; Nosonovsky, M. Small levitating ordered droplet clusters: Stability, symmetry, and Voronoi Entropy. J. Phys. Chem. Lett. 2017, 8, 5599-5602.

20. Weaire, D.; Rivier, N. Soap, cells and statistics-random patterns in two dimensions. Contemporary Phys. 1984, 25, 59-99

21. Descartes, R. Principia Philosophiae; Ludovicus Elzevirius: Amsterdam, The Netherlands, 1644; ISBN 978-90-277-1754-2.

22. Bormashenko, Ed., Frenkel, M., Legchenkova, I. Is the Voronoi Entropy a True Entropy? Comments on "Entropy, Shannon's Measure of Information and Boltzmann's H-Theorem", Entropy 2017, 19, 48, Entropy 2019, 21(3), 251; https://doi.org/10.3390/e21030251

23. Limaye, A.V.; Narhe, R.D.; Dhote, A.M.; Ogale, S.B. Evidence for convective effects in breath figure formation on volatile fluid surfaces. Phys. Rev. Lett. 1996, 76, 3762-3765. 
249

250

251

252

253
24. Martin, C.P., Blunt, M.O., Pauliac-Vaujour, E., Stannard, A., Moriarty, P., Vancea, I., Thiele, U. Controlling pattern formation in nanoparticle assemblies via directed solvent dewetting. Phys. Rev. Lett. 2007, 99, 116103.

25. Zhu, H. X., Thorpe, S. M., Windle, A. H. The geometrical properties of irregular twodimensional Voronoi tessellations, Philosophical Magazine A 2001, 81 (12), 2765-2783. 